\title{
Avaliação curricular na educação superior em odontologia: discutindo as mudanças curriculares na formação em saúde no Brasil
}

\author{
Ramona Fernanda Ceriotti Toassi*, Juliana Maciel de Souza**, Alexandre Baumgarten***, \\ Cassiano Kuchenbecker Rösing****
}

\author{
* Professora Adjunta. Departamento de Odontologia Preventiva e \\ Social. Núcleo de avaliação da unidade. Faculdade de Odontologia, \\ Universidade Federal do Rio Grande do Sul (UFRGS) \\ ** Pedagoga. Técnica em assuntos educacionais. Núcleo de avaliação \\ da unidade. Faculdade de Odontologia, Universidade Federal do \\ Rio Grande do Sul (UFRGS) \\ *** Estudante bolsista de iniciação científica (PROBIC FAPERGS). \\ Faculdade de Odontologia, Universidade Federal do Rio Grande \\ do Sul (UFRGS) \\ **** Professor Associado. Departamento de Odontologia Conservadora. \\ Faculdade de Odontologia, Universidade Federal do Rio Grande \\ do Sul (UFRGS)
}

\section{RESUMO}

O objetivo do estudo foi analisar o desenvolvimento do processo de mudanças curriculares na formação superior em Odontologia em uma Universidade Federal no Sul do Brasil. O método de investigação foi predominantemente qualitativo (estudo de caso). Foram convidados a participar do estudo todos os estudantes do $1^{\circ}$ ao $10^{\circ}$ semestre do curso. A coleta de dados envolveu a análise de documentos e aplicação de questionário semiestruturado. Os dados objetivos foram analisados pelo software estatístico SPSS versão 18.0 e os relatos foram interpretados por meio da análise de conteúdo. Participaram do estudo 360 estudantes (taxa de resposta $88,5 \%$ ), sendo a maioria mulheres $(69,2 \%)$, jovens $(58,1 \%$ com idade entre 17 a 22 anos), solteiros $(96,4 \%)$ e sem filhos $(98,3 \%)$. Grande parte dos estudantes acredita estar recebendo uma sólida formação para atuar no mercado de trabalho e mostrou-se satisfeito com o curso. Como potencialidades, os estudantes destacaram o atual currículo que enfatiza a humanização da saúde e os ganhos na formação com o período dos estágios curriculares supervisionados no Sistema Único de Saúde. Fragilidades foram apontadas em alguns aspectos da integração curricular e em relação ao processo de avaliação das aprendizagens. Recomenda-se a avaliação do currículo, de modo contínuo e formador, permitindo sua transformação/reconstrução no curso de seu desenvolvimento.

\section{DESCRITORES}

Avaliação Curricular. Currículo. Ensino Odontológico. Ensino Superior.

$\mathbf{U}$ m dos aspectos importantes das políticas de educação para o Ensino Superior da última década foi a proposta de flexibilização curricular nos cursos de graduação. ${ }^{24}$

Práticas curriculares foram produzindo e moldando a subjetividade do cirurgião-dentista do mundo contemporâneo e, também, a clínica por ele desempenhada. ${ }^{28}$ No Brasil, com raras exceções, o ensino da Odontologia formava profissionais para atuarem exclusivamente em clínicas particulares e para o exercício privado da profissão, com um perfil de atenção individualizada e com altos custos. Tal formação evidenciava a fragmentação de conteúdos e o processo de ensino-aprendizagem centrado no professor especialista, o que gerava uma excessiva especialização e um distanciamento da preparação 
para o cuidado efetivo e resolutivo em saúde. ${ }^{1}$ Por outro lado, um número cada vez maior de novas faculdades de Odontologia contrastava-se com um panorama nacional de altos índices de prevalência de cárie dentária, doenças periodontais, oclusopatias e de indivíduos edêntulos. ${ }^{19}$

Com a implementação das Diretrizes Curriculares Nacionais (DCN) nos cursos da saúde, incluindo a Odontologia, ${ }^{7}$ houve a flexibilização curricular e a oportunidade das instituições de elaborarem seus projetos pedagógicos voltados a cada realidade local e regional, ao contrário do currículo mínimo adotado nos cursos superiores até então, que inibia a inovação e a criatividade das instituições formadoras com o excesso de detalhamento de conteúdos obrigatórios.

Essa nova proposta de formação prevê o equilíbrio entre a excelência técnica e a relevância social. ${ }^{18}$ As DCN valorizaram a relevância social das ações de saúde e do próprio ensino, o que implicou, necessariamente, na construção de currículos que preparassem o profissional para trabalhar a partir das necessidades da população, num contexto de mudanças no perfil epidemiológico das doenças bucais, adotando um conceito mais ampliado de saúde e de novas práticas baseadas em evidências científicas..$^{20,30}$

Essa mudança na educação superior assumindo a formação de habilidades e competências tem possibilitado avanços importantes em várias instituições de ensino brasileiras, com a implantação de projetos pedagógicos inovadores em direção a uma educação integral e mais humanizada. Por outro lado, esse mecanismo tão aberto e flexível na organização dos currículos, gera preocupações em um país de dimensões continentais como o Brasil. ${ }^{8}$ As orientações das Diretrizes estimulam as escolas a superar as concepções conservadoras, a rigidez, o conteudismo e as prescrições estritas nos currículos mínimos, mas não definem um caminho único. ${ }^{11}$ Assim, é preciso examinar permanentemente a experiência concreta e os rumos de cada instituição. Nesse sentido, o contínuo processo de avaliação da experiência curricular e da trajetória dos estudantes é fundamental.

Após três anos de discussão com a comunidade acadêmica, para atender a essas novas Diretrizes, em 2005, deu-se início a implementação do processo de reestruturação curricular na Faculdade de Odontologia da Universidade Federal do Rio Grande do Sul (FOUFRGS), prevendo um ensino mais integrado às demandas sociais. De 2005 a 2011, quatro turmas já foram formadas a partir dessa proposta.
Abarcado por procedimentos de avaliação, o currículo é mais valorizado, sendo a expressão da concretização de seu significado tanto para os professores quanto para os alunos. ${ }^{22}$

Entendendo a avaliação sobre os componentes curriculares como um dos aspectos da transformação desse currículo, o presente estudo teve por objetivo analisar o desenvolvimento de um processo de reestruturação curricular, na perspectiva de seus estudantes.

\section{METODOLOGIA}

A metodologia utilizada foi o estudo de caso, numa perspectiva de análise predominantemente qualitativa, cujo campo de investigação foi a FOUFRGS.

Baseado e orientado pelas DCN, o curso de Odontologia da UFRGS reestruturou seu currículo por meio de uma construção coletiva entre estudantes, professores e funcionários técnico-administrativos e implementou o novo modelo curricular em 2005, processo este que constituiu uma transformação na história da faculdade. A Faculdade de Odontologia foi criada em 1898, vinculada à Faculdade de Medicina, sendo reconhecida pelo governo Federal em 1900. De 1922 a 1932, o curso foi fechado por razões logísticas, conceituais e estruturais, sendo reaberto durante o Governo de Getúlio Vargas. Em 1952 a faculdade conquistou sua autonomia e desde o ano de 1968, se localiza em sua sede própria. Desde a reabertura do curso houve diversas reformas curriculares, sendo que a última foi feita em 2005. O novo currículo propôs a alteração do perfil do profissional egresso, enfatizando atividades de promoção, preservação e recuperação da saúde da população, norteadas pelos princípios da ética e da bioética. ${ }^{27}$

Assim, foram convidados a participar do estudo todos os estudantes da graduação em Odontologia, do $1^{\circ}$ ao $10^{\circ}$ semestres que tivessem interesse e disponibilidade. A coleta de dados aconteceu pela análise de documento (Projeto Pedagógico do curso de Odontologia) e pela aplicação de questionários semiestruturados, previamente testados.

O questionário utilizado não identificou o estudante e estava dividido em quatro blocos:

- perfil sociodemográfico dos estudantes;

- sobre a opção pela Odontologia e sobre o curso;

- perspectiva de atuação profissional/educação permanente e

- sobre o desenvolvimento curricular. 
Para a análise dos dados de perfil e das respostas referentes às questões objetivas do questionário foi criado um banco de dados digitado no software estatístico SPSS versão 18.0. Já as informações referentes às questões abertas (narrativas, percepções dos estudantes) foram interpretadas seguindo o método da análise de conteúdo. ${ }^{6}$

O estudo foi avaliado e aprovado pelo Comitê de Ética em Pesquisa da UFRGS ( ${ }^{\circ}$ 20297) e todos os participantes assinaram um termo de consentimento livre e esclarecido.

\section{RESULTADOS E DISCUSSÃO}

O currículo está relacionado a tudo o que é planejado, implementado, ensinado, aprendido, avaliado e pesquisado, independentemente do nível ou etapa do sistema educacional. Mais do que uma lista de conteúdos intermináveis, classificados por disciplina, o currículo deve ser entendido como um documento de orientação, uma proposta que estabelece um plano educacional, oferecendo aos alunos conhecimento, atitudes, valores, habilidades e competências valorizadas socialmente..$^{14,17}$

O curso de odontologia da UFRGS, em consonância com os demais cursos de graduação na área da saúde, passou por transformações em suas estruturas curriculares e pedagógicas, buscando atender à demanda de articulação ao Sistema Único de Saúde (SUS), tendo a integralidade da atenção à saúde como eixo norteador dessas mudanças. ${ }^{9}$

As características dos estudantes de Odontologia, suas narrativas e percepções em relação ao processo de reestruturação curricular foram apresentadas em cinco momentos:

- perfil sociodemográfico dos estudantes;

- sobre a opção pela odontologia e sobre o curso;

- perspectiva de atuação profissional/educação permanente;

- sobre o desenvolvimento curricular e

- potencialidades emergentes a partir da mudança curricular.

\section{Perfil sociodemográfico dos estudantes}

Participaram da avaliação curricular 360 estudantes de Odontologia, do $1^{\circ}$ ao $10^{\circ}$ semestre (taxa de resposta de $88,5 \%)$. Destes estudantes, a maioria eram mulheres $(69,2 \%)$, jovens $(58,1 \%$ com idade entre 17 a 22 anos), solteiros (96,4\%), sem filhos $(98,3 \%)$, do estado do Rio Grande do Sul $(88,9 \%)$ e nunca trabalharam (71,4\%). Observar Tabela 1.

A maior parte dos estudantes realizou o ensino
Tabela 1 - Distribuição dos estudantes segundo perfil demográfico.

\begin{tabular}{|c|c|c|c|}
\hline \multicolumn{2}{|r|}{ Variáveis } & $\mathbf{n}$ & $\%$ \\
\hline \multirow{3}{*}{ Sexo } & Feminino & 249 & 69,2 \\
\hline & Masculino & 107 & 29,7 \\
\hline & Não informou & 4 & 1,1 \\
\hline \multirow{6}{*}{ Idade } & 17 a 19 anos & 56 & 15,6 \\
\hline & 20 a 22 anos & 153 & 42,5 \\
\hline & 23 a 25 anos & 116 & 32,2 \\
\hline & 26 a 28 anos & 29 & 8,1 \\
\hline & 29 a 33 anos & 3 & 0,8 \\
\hline & Não informou & 3 & 0,8 \\
\hline \multirow{3}{*}{$\begin{array}{l}\text { Estado } \\
\text { civil }\end{array}$} & Solteiro & 347 & 96,4 \\
\hline & Casado & 11 & 3,0 \\
\hline & Não informou & 2 & 0,6 \\
\hline \multirow{2}{*}{$\begin{array}{c}\text { Presença } \\
\text { de filhos }\end{array}$} & Sim & 6 & 1,7 \\
\hline & Não & 354 & 98,3 \\
\hline \multirow{9}{*}{$\begin{array}{l}\text { Estado de } \\
\text { origem }\end{array}$} & Rio Grande do Sul & 320 & 88,9 \\
\hline & Mato Grosso do Sul & 2 & 0,6 \\
\hline & Minas gerais & 1 & 0,3 \\
\hline & São paulo & 5 & 1,3 \\
\hline & Paraná & 2 & 0,5 \\
\hline & Santa catarina & 6 & 1,7 \\
\hline & Rio de Janeiro & 2 & 0,6 \\
\hline & Outro país & 2 & 0,6 \\
\hline & Não informou & 20 & 5,5 \\
\hline \multirow{4}{*}{$\begin{array}{c}\text { Já } \\
\text { trabalhou }\end{array}$} & Sim, trabalha no momento & 22 & 6,1 \\
\hline & $\begin{array}{l}\text { Sim, trabalhou antes do } \\
\text { curso começar }\end{array}$ & 67 & 18,6 \\
\hline & $\begin{array}{l}\text { Sim, durante o curso, mas } \\
\text { agora não trabalha mais }\end{array}$ & 14 & 3,9 \\
\hline & Não, nunca trabalhou & 257 & 71,4 \\
\hline & Total & 360 & 100 \\
\hline
\end{tabular}

fundamental e médio em escolas da rede privada de ensino ( $56,9 \%$ e $67,2 \%$, respectivamente) e curso pré-vestibular antes do ingresso na universidade $(94,4 \%)$. Para $80,3 \%$, a Odontologia foi o primeiro curso de graduação iniciado.

Em relação à família dos estudantes, $56,4 \%$ dos pais e $51,4 \%$ das mães possuíam o ensino superior completo. A maior parte dos pais estava trabalhando ( $61,4 \%$ das mães e $72,8 \%$ dos pais). Mais da metade dos estudantes $(50,3 \%)$ relatou ter renda familiar entre 6 a 15 salários mínimos. O pai foi o responsável pelo maior ganho familiar $(58,9 \%)$. A presença de dentista na família foi relatada por $32,2 \%$ dos estudantes. 
Cerca de metade dos estudantes relataram vínculo com projetos de pesquisa, extensão ou monitoria acadêmica (remunerados ou voluntários).

\section{Sobre a opção pela odontologia e sobre o curso}

Quando optaram pelo curso, $45,8 \%$ dos estudantes estavam absolutamente decididos pela Odontologia, escolhendo-a por interesse pessoal, por ser da área da saúde e pela influência de amigos e da família.

"Porque reconheci no curso várias áreas de atuação de meu interesse. Encontrei na odontologia a união de trabalhar o lado humano junto com a oportunidade de melhorar a qualidade de vida dos pacientes". (Estudante 46)

Sobre as expectativas em relação ao curso de Odontologia, as seguintes categorias emergiram das respostas dos estudantes:

- formação de qualidade;

- realização pessoal e profissional;

- formação para o mercado de trabalho;

- retorno financeiro;

- formação voltada à demanda da população e

- aprendizagem.

\footnotetext{
"Minha expectativa é que o curso amplie minha visão sobre a Odontologia, bem como me forneça conhecimento necessário para a realização das técnicas odontológicas (para que eu entenda as técnicas e saiba o porquê de estar aplicando-as a determinado paciente)". (Estudante 106)
}

Grande parte dos estudantes (72,2\%) se mostrou satisfeita com o curso e 85,3\% acreditam que estão recebendo uma sólida formação para atuar no mercado de trabalho.

As maiores ênfases do curso de Odontologia, segundo a percepção dos estudantes, foram Saúde Pública/Saúde Coletiva/SUS, seguidos por disciplinas específicas e formação com ênfase no humano/saúde como um todo.

\section{Perspectiva de atuação profissional/ educação permanente}

Após a graduação, 50,3\% dos estudantes pretendem trabalhar no setor público e privado, mas demonstraram pouco interesse em atuar exclusivamente no serviço público $(1,1 \%)$. Essa informação corrobora achados do estudo de Rösing et al. (2009), ${ }^{21}$ avaliando quatro currículos odontológicos, sendo dois no Brasil e dois na Noruega.
Além disso, os estudantes pretendem se especializar $(97,5 \%)$, sendo que $52 \%$ planejam iniciar a especialização de 6 meses até 1 ano após o término do curso. As áreas de especialidade mais citadas foram a prótese/implantodontia, a cirurgia e a ortodontia.

\section{Sobre o desenvolvimento curricular}

Quando optaram pelo curso de Odontologia, a maior parte dos estudantes não sabia sobre sua proposta curricular $(78,6 \%)$ e relataram que esta foi apresentada ao chegarem à Faculdade $(81,4 \%)$. Atualmente, $53,6 \%$ dos estudantes afirmaram conhecer bem a estrutura curricular do curso, mas em relação ao projeto pedagógico $36,9 \%$ relata apenas ter ouvido falar e $16,7 \%$ relatou não conhecer e não saber seu conteúdo. Em relação aos planos de ensino, $48,9 \%$ dos estudantes afirmaram que estes foram apresentados por todas as disciplinas (Tabela 2).

$\mathrm{O}$ uso de metodologias que permitem a participação do estudante no processo de ensino-aprendizagem foi percebido especialmente por meio de seminários, atividades práticas, discussão de casos clínicos e nos estágios (25,3\%), em disciplinas específicas $(12,5 \%)$ e na participação das aulas e leituras prévias sobre o conteúdo teórico $(8,9 \%)$. Atividades de pesquisa, estágio e monitorias também foram citadas pelos estudantes (Tabela 2).

Segundo o artigo 13 das DCN, ${ }^{7}$ a estrutura do curso de graduação em Odontologia deve explicitar a definição do perfil a ser formado, aproximar o conhecimento básico da sua utilização clínica e utilizar metodologias de ensino-aprendizagem que permitam a participação ativa dos alunos neste processo.

A adoção de novos métodos de ensino-aprendizagem constitui um caminho inovador para a formação profissional em saúde onde o estudante tem a possibilidade de ressignificar suas aprendizagens, assumindo um papel cada vez mais ativo, desenvolvendo a capacidade de construir conhecimento com autonomia, refletir sobre problemas reais e formulação de ações originais e criativas, potencialmente capazes de transformar a realidade social. ${ }^{13,16}$

Ainda que as metodologias ativas tenham sido identificadas pelos estudantes, a adoção de novas estratégias metodológicas foram sugeridas.

\footnotetext{
“[...] a presença de seminários busca isso. Porém, na nossa

formação houve exagero desse recurso, sendo importante

buscar outros métodos". (Estudante 240)
}

Mais do que mudanças aritméticas de créditos na 
Tabela 2 - Desenvolvimento curricular na perspectiva dos estudantes.

\begin{tabular}{|c|c|c|c|}
\hline \multicolumn{2}{|r|}{ Variáveis } & $\mathbf{n}$ & $\%$ \\
\hline \multirow{3}{*}{$\begin{array}{l}\text { Conhecimento da } \\
\text { proposta curricular }\end{array}$} & Conhecia a proposta & 76 & 21,1 \\
\hline & Não conhecia a proposta & 283 & 78,6 \\
\hline & Não informou & 1 & 0,3 \\
\hline \multirow{3}{*}{$\begin{array}{l}\text { Apresentação da } \\
\text { proposta curricular }\end{array}$} & Sim, a proposta foi apresentada & 293 & 81,4 \\
\hline & Não, a proposta não foi apresentada & 62 & 17,2 \\
\hline & Não lembrava & 5 & 1,4 \\
\hline \multirow{4}{*}{$\begin{array}{l}\text { Conhecimento da } \\
\text { organização curricular }\end{array}$} & Conhece bem & 193 & 53,6 \\
\hline & Conhece um pouco & 164 & 45,6 \\
\hline & Não conhece & 2 & 0,5 \\
\hline & Não informou & 1 & 0,3 \\
\hline \multirow{5}{*}{$\begin{array}{l}\text { Conhecimento do } \\
\text { projeto pedagógico }\end{array}$} & Conhece bem & 43 & 11,9 \\
\hline & Já leu algumas coisas & 123 & 34,2 \\
\hline & Apenas ouviu falar & 133 & 36,9 \\
\hline & Não conhece e não sabe qual seu conteúdo & 60 & 16,7 \\
\hline & Não informou & 1 & 0,3 \\
\hline \multirow{5}{*}{ Planos de ensino } & Foram apresentados por todas as disciplinas & 176 & 48,9 \\
\hline & Foram apresentados apenas por algumas disciplinas & 162 & 45,0 \\
\hline & Não foram apresentados & 7 & 1,9 \\
\hline & Não lembrava & 12 & 3,4 \\
\hline & Não informou & 3 & 0,8 \\
\hline \multirow{8}{*}{$\begin{array}{l}\text { Uso de metodologias } \\
\text { ativas }\end{array}$} & Percebe em seminários, atividades práticas, discussão de casos e nos estágios & 91 & 25,3 \\
\hline & Percebe, em disciplinas específicas & 45 & 12,5 \\
\hline & Percebe pela participação do aluno nas aulas e leituras prévias & 32 & 8,9 \\
\hline & Percebe pela participação em atividades de monitoria, iniciação científica, PET & 7 & 1,9 \\
\hline & Percebe nas pesquisas de satisfação do aluno e avaliação da universidade & 7 & 1,9 \\
\hline & Percebe, mas não informou como & 12 & 3,3 \\
\hline & Não percebe ou percebe pouco espaço para a participação do estudante & 105 & 29,2 \\
\hline & Não informou & 61 & 17,0 \\
\hline \multicolumn{2}{|r|}{ Total } & 360 & 100 \\
\hline
\end{tabular}

grade horária e de seus valores, as mudanças necessárias nas reformulações curriculares passam por questões que dizem respeito ao conteúdo pretendido e às estratégias de ensino e de aprendizagem. ${ }^{3}$

Sobre a integração entre as disciplinas, as $\mathrm{DCN}^{7}$ estabelecem-na como condição essencial em todo o processo de ensino-aprendizagem. Na FOUFRGS, 99,4\% dos estudantes percebem a integração entre as disciplinas, principalmente durante o período dos estágios curriculares supervisionados que acontecem no último ano do curso e $43,3 \%$ a perceberam entre os conhecimentos das ciências básicas e sociais.

\footnotetext{
"[...] nos é enfatizado durante o curso a visão humanística e integral do paciente, sempre levando em conta sua rela-
}

ção fisiológica com o ambiente.” (Estudante 234)
"[...] a integração foi bastante visível, inclusive nos debates em sala de aula, e o conhecimento foi sendo construído a cada etapa, o que facilitou a visão integral da saúde públi- ca”. (Estudante 248)

As ações integrativas auxiliam o estudante a construir um quadro teórico-prático global mais significativo e mais próximo dos desafios presentes na realidade profissional dinâmica e única, na qual atuará depois de concluída a graduação. ${ }^{2} \mathrm{O}$ ensino baseado na integração proporciona uma aprendizagem mais estruturada e rica, pois os conhecimentos estão organizados em torno de estruturas conceituais e meto- 
dológicas compartilhadas por várias disciplinas. ${ }^{5}$

Essa integração curricular, no entanto, está em processo de construção e desafios foram verificados em relação à efetivação da proposta.

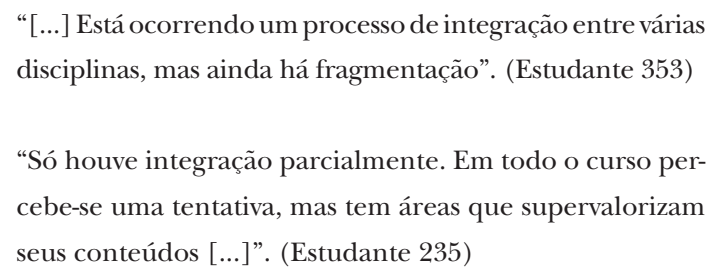

Essas mesmas dificuldades quanto à integração foram verificadas por estudantes e professores em estudo sobre a análise do processo de mudança curricular no curso de Odontologia em uma Universidade Comunitária no Sul do Brasil. ${ }^{26}$

Sobre o processo de avaliação das aprendizagens, de modo geral, os estudantes entendem as avaliações como positivas, efetivas, coerentes, válidas, preparando-os para o próximo semestre, exigentes e rigorosas.

Fragilidades foram apontadas no que se referiu à falta de clareza nos critérios das avaliações e ao excesso de conteúdo cobrado.

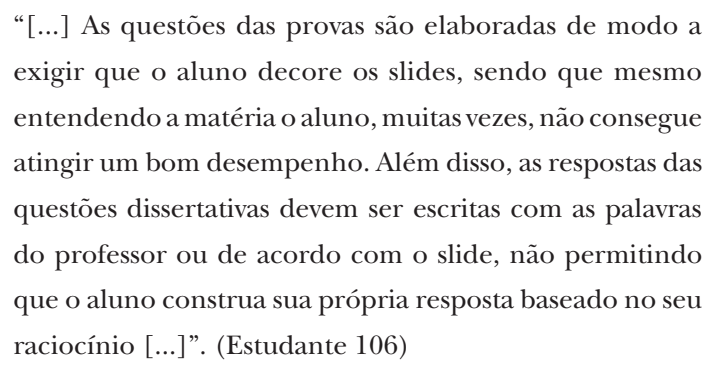

A avaliação das aprendizagens continua a marcar uma presença relevante e muitas vezes polêmica no centro dos grandes debates da educação atual, pois é um processo que tem profundas implicações na organização e no funcionamento dos sistemas educacionais e das instituições de ensino, na forma pela qual os professores organizam seu ensino ou no desenvolvimento das aprendizagens por parte dos estudantes. ${ }^{10}$

As avaliações dos alunos devem ter como referência as diretrizes curriculares ${ }^{7}$ e basearem-se nas competências, habilidades e conteúdos curriculares desenvolvidos. Tanto as competências a serem adquiridas quanto os indicadores de desempenho e resultados esperados devem estar claramente definidos, descritos e disponibilizados para todos os envol- vidos no processo educacional..$^{23}$

\section{Potencialidades emergentes a partir da mudança curricular}

Os estudantes referiram-se ao atual currículo voltado à humanização da saúde como uma de suas potencialidades, mostrando preocupação no sentido de terem uma formação voltada para o atual mercado de trabalho do país.

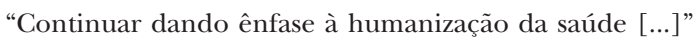

"[...] Aproximar cada vez mais o curso da realidade da saúde da população e do mercado de trabalho disponível”. (Estudante 279)

Além disso, o período dos estágios curriculares supervisionados no SUS do último ano do curso foi um dos destaques positivos da reestruturação curricular. Os estudantes do último semestre assim se manifestaram em relação aos estágios curriculares:

\footnotetext{
"Importantes para conhecer a realidade prática e teórica do sistema público de saúde. Oferecem mais oportunidade para o desenvolvimento da autonomia no atendimento ao paciente”. (Estudante 236)
}

A aprendizagem nos serviços públicos de saúde é fundamental para a formação em saúde, possibilitando a aproximação com a comunidade pela realização de atividades que vão além dos limites físicos da instituição formadora. ${ }^{4,29}$

Os espaços de interseção entre serviços e ensino são de grande importância para a formação em saúde e para a consolidação do SUS. A integração ensino-serviço é um dos eixos que busca solidificar a proposta curricular, por meio da diversidade de ações na interface entre o serviço e o serviço. ${ }^{25}$

Outra questão entendida como importante pelos estudantes referiu-se a sua participação na melhoria da Faculdade (ouvir opinião, melhorar o diálogo) e na sugestão de continuidade do processo de avaliação.

\footnotetext{
"Criar diálogo com os alunos. Romper barreiras de comunicação. Incentivar os alunos a melhorar a faculdade". (Estudante 140)
}

Por fim, os estudantes sugeriram que o currículo seja avaliado continuamente, permitindo a trans- 
formação/reconstrução no curso de seu desenvolvimento.

Uma proposta curricular inovadora está associada com mudanças relacionadas à perspectiva de seus estudantes ${ }^{15}$ e a transformações na cultura pedagógica da instituição, tão necessárias para um efetivo currículo integrado. Tudo isso só será possível se as avaliações curriculares continuarem acontecendo. ${ }^{12}$

\section{CONCLUSÃO}

Dos 360 estudantes que participaram desse estudo, a maioria eram mulheres, jovens, do estado do Rio grande do Sul, solteiros, sem filhos e que nunca trabalharam. Quando optaram pelo curso, 45,8\% dos estudantes estavam absolutamente decididos e 85,3\% acreditam estar recebendo uma sólida formação para atuar no mercado de trabalho. Após a graduação, $50,3 \%$ das estudantes pretendem trabalhar no setor público e privado e $97,5 \%$ querem se especializar, de 6 meses a 1 ano após o término do curso (52\%), especialmente nas áreas de prótese/implantandontia, cirurgia e ortodontia.

Os estudantes apontaram como potencialidades, o atual currículo que enfatiza a humanização da saúde e o período dos estágios curriculares no SUS. Desafios foram verificados na efetivação da integração entre as disciplinas e também em relação ao processo de avaliação das aprendizagens.

Entende-se que mudanças curriculares são processuais e precisam de tempo para que os avanços pretendidos possam se concretizar. Recomenda-se, assim, a avaliação do currículo, de modo contínuo e formador.

A temática pesquisada não se esgota com os resultados deste estudo, mas abre oportunidade para a realização de pesquisas futuras com diferentes atores do processo e Instituições de Ensino Superior no país, trazendo contribuições pertinentes para a discussão sobre currículos integrados e inovadores na área da saúde.

\section{ABSTRACT}

\section{Curricular evaluation of higher education in dentistry: a discussion about the effects of curriculum changes on health care training in Brazil}

The aim of this study was to analyze the effects of the process of curricular change implemented in the dental school of a federal university in southern Brazil. The research method was mostly qualitative (case study). All students from the $1^{\text {st }}$ to the $10^{\text {th }}$ se- mester were invited to participate. Data collection involved analysis of documents and application of a semi-structured questionnaire. Data were analyzed using SPSS 18.0 statistical software, and the reports were interpreted by content analysis. A total of 360 students participated in the study $(88.5 \%$ response rate), most of whom were females $(69.2 \%)$, young adults (58.1\% aged 17 to 22 years), single (96.4\%) and without children (98.3\%). A significant part of the students think that they are receiving a sound education that will allow them to enter the job market and are satisfied with their course. Among the potential benefits, students highlighted the current curriculum that emphasizes the humanization of healthcare and improvements in their training as a result of supervised internships in the Public Health System. Some weaknesses were identified in respect to parts of the curricular integration and also to the process of student performance assessment. Continuous and integrated evaluation of the institution's curriculum is recommended to allow it to be transformed and reconstructed as it is developed.

\section{DESCRIPTORS}

Curricular Evaluation. Curriculum. Dental Education. Education, Higher. -

\section{REFERÊNCIAS}

1. Albuquerque VS, Batista RS, Tanji S, Moço ETM. Currículos disciplinares na área de saúde: ensaio sobre saber e poder. Interface comun. saúde educ. 2009 out/dez; 13(31):261-72.

2. Anastasiou LGC. Da visão de ciência à organização curricular. In:Anastasiou LGL, Alves LP. Processos de ensinagem na universidade: pressupostos para as estratégias de trabalho em aula. 5.ed. Joinville: UNIVILLE, 2005; p.40-63.

3. Araújo ME, Zilbovicius C. A formação para o trabalho no Sistema Único de Saúde (SUS). In: Moysés ST, Kriger L, Moysés SJ (Coord.). Saúde bucal das Famílias: trabalhando com evidências. São Paulo: Artes Médicas; 2008. p.277-90.

4. Aston-Brown RE, Branson B, Gadbury-Amyot CC, Bray KK. Utilizing Public Health Clinics for Service-Learning Rotations in Dental Hygiene: A Four-Year Retrospective Study. J Dent Educ. 2009 mar/jun; 73(3):358-74.

5. Audy JLN. Interdisciplinaridade e complexidade na construção do conhecimento: o desafio da integração ensino-pesquisa. In: Engers MEA, Morosini MC (Org.). Pedagogia universitária. Porto Alegre: EDIPUCRS; 2007. p.31-37.

6. Bardin, L. Análise de conteúdo.70 ed. São Paulo: Almedina; 2011.

7. Brasil. Resolução CNE/CNS 3/2002, de 4 de março de 2002. Diretrizes Curriculares Nacionais do Curso de Odontologia. 
Diário Oficial da União, Ministério da Saúde e Ministério da Educação, Brasília, DF. Seção 1, p.10.

8. Carvalho ACP. Planejamento do curso de graduação de Odontologia. Rev. ABENO. 2004; 4(1):7-13.

9. Ceccim RB, Feuerwerker LCM. O Quadrilátero da Formação para a Área da Saúde: Ensino, Gestão, Atenção e Controle Social. Physis (Rio J.). 2004; 14(1):41-65.

10. Fernandes D. Avaliação, aprendizagens e currículo: para uma articulação entre investigação, formação e práticas. In: Barbosa RLL (Org.). Formação de Educadores. São Paulo: UNESP; 2006. p.15-36.

11. Feuerwerker LCM, Almeida M. Diretrizes Curriculares e projetos pedagógicos: é tempo de ação! Rev. ABENO. 2004; 4(1):14- 6 .

12. Gabriel M, Tanaka EE. Formação profissional em odontologia: percepção discente da interação curricular. Rev. ABENO. 2011; 11(1):19-22.

13. Gomes MPC, Ribeiro VMB, Monteiro DM, Leher EMT, Louzada RCR. O uso de metodologias ativas no ensino de graduação nas ciências sociais e na saúde - avaliação dos estudantes. Ciênc. educ. (Bauru). 2010; 16(1):198-98.

14. Gómez AIP. Competências ou pensamento prático? A construção dos significados de representação e de ação. In: Sacristán JG, Gómez AIP, Rodrígues JBMR, Rasco FA, Méndez JMA. Educar por competências: o que há de novo? Porto Alegre: Artmed; 2011. p.64-114.

15. Junges R, Stello RS, Rösing CK, Samuel SMW. Impact of new curriculum implementation on student experience in a school of dentistry in Brazil. Braz Oral Res. 2011 nov/dez; 25(6):478-84.

16. Marin MJS, Lima EFG, Paviotti AB, Matsuyama DT, Silva LKD, Gonçalvez C. et al. Aspectos das fortalezas e fragilidades no uso das Metodologias ativas de Aprendizagem. Rev. bras. educ. méd. 2010; 34(1):13-20.

17. Mckernan J. Currículo e Imaginação: teoria do processo, pedagogia e pesquisa-ação. Porto Alegre: Artmed; 2009.

18. Morita MC, Kriger L. Mudanças nos cursos de Odontologia e a interação com o SUS. Rev. ABENO. 2004; 4 (1):17-21.

19. Morita MC, Kriger L, Carvalho ACP, Haddad AE. Implantação das Diretrizes Curriculares Nacionais em Odontologia, Maringá: Dental Press, ABENO, OPAS, MS; 2007.
20. Morita MC, Haddad AE. Interfaces da área da Educação e da Saúde na perspectiva da formação e do trabalho das equipes de Saúde da Família. In: Moysés ST, Kriger L, Moysés SJ (Coord). Saúde bucal das famílias: trabalhando com evidências. São Paulo: Artes Médicas; 2008. p.268-76.

21. Rösing CK, Silva DT, Deon P, Oppermann RV, Gjermo P. Avaliação de 4 currículos de odontologia baseada em expectativas e satisfação de alunos - relato de experiências norueguesas e brasileira. Rev. ABENO. 2009; 9 (2):88-94.

22. Sacristán JG. O currículo: uma reflexão sobre a prática. 3.ed. Porto Alegre: Artmed; 2000.

23. Santos WS. Organização curricular baseada em competência na educação médica. Rev. bras. educ. méd. 2011; 35 (1):86-92.

24. Senna MIB, Lima MLR. Diretrizes curriculares nacionais para o ensino de graduação em odontologia: uma análise dos artigos publicados na revista da ABENO, 2002-2006. Arq. odontol. $2009 \mathrm{jan} / \mathrm{mar} ;$ 45(1):30-6.

25. Souza AL, Carcereri DL. Estudo qualitative da integração ensino-serviço em um curso de graduação em odontologia. Interface comun. saúde educ. 2011 out/dez; 15(39):1071-84.

26. Toassi RFC, Stobäus CD, Mosquera JJM, Moysés SJ. Currículo integrado no ensino de Odontologia: novos sentidos para a formação na área da saúde. Interface comun. saúde educ. 2012 jan/mar; 16(40).

27. Universidade Federal do Rio Grande do Sul. Faculdade de Odontologia. Projeto Político Pedagógico. Porto Alegre; 2005.

28. Warmling, CM, Marzola, NR, Botazzo C. Da autonomia da boca: práticas curriculares e identidade profissional na emergência do ensino brasileiro da odontologia. Hist. ciênc. saúde-Manguinhos. 2012 jan/mar; 19 (1):181-95.

29. Werneck MAF, Barreiros SMI, Maia DM, Dutra LS. Nem tudo é estágio: contribuições para o debate. Ciênc. saúde coletiva. 2010 jan; 15(1):221-31.

30. Zilbovicius C, Araujo ME, Botazzo C, Frias AC, Junqueira SR, Junqueira CR. A paradigm shift in predoctoral dental curricula in brazil: evaluating the process of change. J Dent Educ. 2011 abr; $75(4): 557-64$.
Recebido em 08/10/2012

Aceito em 10/12/2012 